\title{
The neural underpinnings of music listening under different attention conditions
}

\author{
Jäncke, Lutz ; Leipold, Simon ; Burkhard, Anja
}

\begin{abstract}
Most studies examining the neural underpinnings of music listening have no specific instruction on how to process the presented musical pieces. In this study, we explicitly manipulated the participants' focus of attention while they listened to the musical pieces. We used an ecologically valid experimental setting by presenting the musical stimuli simultaneously with naturalistic film sequences. In one condition, the participants were instructed to focus their attention on the musical piece (attentive listening), whereas in the second condition, the participants directed their attention to the film sequence (passive listening). We used two instrumental musical pieces: an electronic pop song, which was a major hit at the time of testing, and a classical musical piece. During music presentation, we measured electroencephalographic oscillations and responses from the autonomic nervous system (heart rate and high-frequency heart rate variability). During passive listening to the pop song, we found strong event-related synchronizations in all analyzed frequency bands (theta, lower alpha, upper alpha, lower beta, and upper beta). The neurophysiological responses during attentive listening to the pop song were similar to those of the classical musical piece during both listening conditions. Thus, the focus of attention had a strong influence on the neurophysiological responses to the pop song, but not on the responses to the classical musical piece. The electroencephalographic responses during passive listening to the pop song are interpreted as a neurophysiological and psychological state typically observed when the participants are 'drawn into the music'.
\end{abstract}

DOI: https://doi.org/10.1097/WNR.0000000000001019

Posted at the Zurich Open Repository and Archive, University of Zurich

ZORA URL: https://doi.org/10.5167/uzh-151056

Journal Article

Published Version

Originally published at:

Jäncke, Lutz; Leipold, Simon; Burkhard, Anja (2018). The neural underpinnings of music listening under different attention conditions. NeuroReport, 29(7):594-604.

DOI: https://doi.org/10.1097/WNR.0000000000001019 


\title{
The neural underpinnings of music listening under different attention conditions
}

\author{
Lutz Jäncke $^{\mathrm{a}, \mathrm{b}, \mathrm{c}, \mathrm{d}}$, Simon Leipold ${ }^{\mathrm{a}}$ and Anja Burkhard ${ }^{\mathrm{a}}$
}

\begin{abstract}
Most studies examining the neural underpinnings of music listening have no specific instruction on how to process the presented musical pieces. In this study, we explicitly manipulated the participants' focus of attention while they listened to the musical pieces. We used an ecologically valid experimental setting by presenting the musical stimuli simultaneously with naturalistic film sequences. In one condition, the participants were instructed to focus their attention on the musical piece (attentive listening), whereas in the second condition, the participants directed their attention to the film sequence (passive listening). We used two instrumental musical pieces: an electronic pop song, which was a major hit at the time of testing, and a classical musical piece. During music presentation, we measured electroencephalographic oscillations and responses from the autonomic nervous system (heart rate and highfrequency heart rate variability). During passive listening to the pop song, we found strong event-related synchronizations in all analyzed frequency bands (theta, lower alpha, upper alpha, lower beta, and upper beta). The neurophysiological responses during attentive listening to the pop song were similar to those of the classical musical
\end{abstract}

piece during both listening conditions. Thus, the focus of attention had a strong influence on the neurophysiological responses to the pop song, but not on the responses to the classical musical piece. The electroencephalographic responses during passive listening to the pop song are interpreted as a neurophysiological and psychological state typically observed when the participants are 'drawn into the music'. NeuroReport 29:594-604 Copyright (C) 2018 Wolters Kluwer Health, Inc. All rights reserved.

NeuroReport 2018, 29:594-604

Keywords: attention, electroencephalographic oscillations, heart rate variability, music listening

aDivision of Neuropsychology, Department of Psychology, ' International Normal Aging and Plasticity Imaging Center (INAPIC), 'University Research Priority Program (URPP), Dynamic of Healthy Aging, University of Zurich, Zurich, Switzerland and dDepartment of Special Education, King Abdulaziz University, Jeddah, Kingdom of Saudi Arabia

Correspondence to Lutz Jäncke, PhD, Division of Neuropsychology, Department of Psychology, University of Zurich, Binzmuehlestrasse 14/Box 25, CH-8050 Zurich, Switzerland

Tel: + 4144635 7557; fax: + 4144635 7409; e-mail: lutz.jaencke@uzh.ch

Received 13 February 2018 accepted 5 March 2018

\section{Introduction}

During the past 30 years, many studies have been published examining the neural underpinnings of music listening. Functional MRI and PET studies have shown that music listening is associated with hemodynamic responses in a distributed network. This network comprises the auditory cortex (as a core region with the primary and the secondary auditory cortex), brain regions associated with the control of attention (e.g. superior and inferior parietal cortex as well as areas in the frontal pole), memory (e.g. hippocampus, medial, and inferior temporal cortex), working memory (e.g. frontal cortex), emotion (limbic system including the nucleus accumbens), and motor control (e.g. supplementary motor area and presupplementary motor area) [1-7]. Several electroencephalography (EEG) studies have complemented these findings, either by estimating the intracortical sources on the basis of surface EEG measures [8-10], by uncovering specific functional network characteristics during music listening [11-20], or by reporting increased alpha band power during musical imagery and listening [21,22]. Most of the neuroscientific studies investigating music listening presented the music without specific instructions. However, it is well known from studies using tightly controlled auditory stimuli that the direction and quality of attention influence the brain activation in response to the incoming stimuli [23-26]. In addition, a few music listening studies have shown that brain activations during music listening depend on context effects [27,28]. Thus, there is a need for the study of the neural underpinnings of music listening under different listening conditions. To examine whether attentive or passive listening to music induces different responses in the EEG and the autonomic nervous system (ANS), we designed this exploratory experiment by measuring EEG oscillations, heart rate (HR), and highfrequency heart rate variability (HF-HRV) during different music listening conditions. The musical pieces were presented simultaneously with naturalistic film sequences. In one condition, the participants focused their attention on the music (attentive listening), whereas in the other condition, the focus of attention was directed to the simultaneously presented film sequence (passive listening). Furthermore, we examined whether musical pieces of two different genres evoke diverging EEG and ANS responses in these listening conditions. We used two instrumental musical pieces, one of which was well known to the participants because it was a major hit at the time of testing and the other a classical instrumental piece that has been used in many experiments, but was not well known to the participants. 
As this study was designed explicitly as an exploratory study, we only defined general study questions and no specific hypotheses.

(1) Are the different listening conditions (attentive vs. passive) associated with different EEG oscillatory activation patterns?

(2) Are the EEG responses different for the two musical pieces, especially in the context of the different listening conditions?

(3) Are the different listening conditions (attentive vs. passive) associated with different ANS responses?

\section{Participants and methods Sample}

Thirty individuals ( 25 women and five men) took part in the study. The participants were students enrolled in Psychology at the University of Zurich or in Arts at the Zurich University of the Arts. The mean age of the participants was 22.2 years (range $=18-33$ years). All participants were consistently right-handed according to the Annett Handedness Questionnaire [29]. None of the participants reported any present or past psychiatric, neurological, or audiological disorders. All participants denied consumption of illegal drugs and regular medication. The participants' history of musical training was assessed using an in-house questionnaire. Twenty-eight of the participants played an instrument or took singing lessons during childhood or youth. On average, those participants started playing when they were 8.3 years old and continued to play regularly for 7.3 years. Twenty-six of the participants reported listening to pop music and 11 to classical music. The participants received course credits or monetary compensation for participation. Written informed consent was obtained from the participants before participation. The study was approved by the Ethics Committee of the University of Zurich and carried out following the principles of the Declaration of Helsinki.

\section{Stimulus material}

The stimuli consisted of two musical pieces from different genres (classical and electronic pop music) and two naturalistic film sequences. The two musical stimuli were (i) Sonate für zwei Klaviere in D-Dur, Allegro con spirito, KV 448 (375a) by Wolfgang A. Mozart, performed by Yaara Tal \& Andreas Groethuysen and (ii) Sonnentanz by the electronic pop music group Klangkarussell. The preprocessing of the auditory stimuli was performed using Audacity software (version 1.3.14 Beta, http://www.audacity.de/) and included the following steps: first, the amplitude of the musical pieces was normalized. Second, only the first $4 \mathrm{~min}$ of each musical piece was kept, with the rest of the piece being cut out. Third, a linear fade-in was applied to the first $500 \mathrm{~ms}$ and a linear fade-out was applied to the last $2000 \mathrm{~ms}$ of each musical piece. The two musical pieces were chosen because they differ in several aspects including acoustic features and publicity level. The musical piece Sonnentanz is a well-known musical piece, which was on the Swiss hit charts for several months, whereas the Mozart piece was not well known to most of the examined participants. In addition, both pieces differ markedly in terms of major acoustic features (intensity variation: Sonnentanz $=55 \%, \mathrm{KV}$ $448=70 \%$; spectral entropy: Sonnentanz $=0.71$, KV $448=0.64$; spectral flatness: Sonnentanz $=0.20$, KV $448=0.014)$. For the calculation of these acoustic features, we used the toolbox seewave (version 2.0.5, http://rug.mnhn.fr/seewave) in R. The two visual stimuli were taken from a YouTube video showing a train ride from the driver's perspective. The preprocessing of the visual stimuli was performed using iMovie software (version 10.1, http://www.apple.com/de/imovie/) and Windows Movie Maker 2012. First, the audio track of the video was removed. Second, the video was decolorized. Third, two nonoverlapping sequences of $4 \mathrm{~min}$ each were taken from the video, and finally, a visual fade-in effect was applied to the first $1500 \mathrm{~ms}$ of each sequence.

\section{Experimental procedure}

The participants were seated in a comfortable chair in a sound-shielded room with electromagnetic isolation. Before the experiment, 8 min of resting-state EEG and electrocardiography (ECG) were acquired: $4 \mathrm{~min}$ with eyes open (EO) and 4 min with eyes closed. For the EO condition, participants were instructed to look at a fixation cross, relax, and let their mind wander. The EO condition was used as a baseline condition for the subsequent experimental conditions. After the resting state, participants performed a task in which they simultaneously listened to musical pieces and watched film sequences while EEG and ECG were acquired. The musical stimuli were presented using in-ear headphones (Sennheiser, Wedemark, Germany) at a comfortable sound pressure level. The film stimuli were presented on a $19^{\prime}$ screen (Belinea, Wittmund, Germany). Stimulus presentation was controlled by Presentation software (version 17.1, http://www.neu robs.com/).

The experiment comprised four conditions, which were presented in a randomized order. In the Mozart-Attentive condition, the musical piece by Mozart was presented simultaneously with one of the film sequences for $4 \mathrm{~min}$, and the participants were instructed to attend the music by counting the occurrence of certain musical aspects (pauses, changes in loudness). In the Klangkarussell-Attentive condition, the musical piece by Klangkarussell was presented simultaneously with the other film sequence (i.e. the sequence that was not used in the Mozart-Attentive condition). The 
instructions for the Klangkarussell-Attentive condition were the same as in the Mozart-Attentive condition. In the Mozart-Passive condition, the physical stimulation was identical to the Mozart-Attentive condition. However, in the Mozart-Passive condition, the participants were instructed to focus their attention on the film sequence by counting the occurrence of certain visual aspects (railway signals, railway switches). The physical stimulation of the Klangkarussell-Passive condition was identical to the Klangkarussell-Attentive condition, with the instructions being the same as in the Mozart-Passive condition. The combination of the musical pieces with the specific film sequence was fixed within each participant, but counterbalanced across participants. The instructions were explained to the participants in detail before the experiment. After each condition, the participants rated the valence and arousal induced by the musical pieces on a seven-point Likert scale. In addition, the participants rated the subjectively experienced difficulty to focus their attention on a seven-point Likert scale.

\section{Electroencephalography data acquisition and preprocessing}

EEG data were recorded using a high-density electrode net with 128 channels (HydroCel GSN 130; Electrical Geodesics, Eugene, Oregon, USA) in combination with an EEG amplifier (Net Amps 300; Electrical Geodesics) and Net Station software (version 4.4, http://www.egi.com/ clinical-division/net-station). The sampling rate of the EEG data was $500 \mathrm{~Hz}$, electrode $\mathrm{Cz}$ was used as the online reference electrode, and an online bandpass filter of baseline condition. Then, the data were again segmented into segments of $2 \mathrm{~s}$ length.

\section{Electroencephalography data analysis}

Spectral analysis of the EEG data was carried out in Brain Vision Analyzer 2. For each $2 \mathrm{~s}$ segment, a frequency spectrum was calculated by a fast Fourier transformation, in which the segments were tapered with a Hanning window. The frequency resolution of the resulting spectra was $0.5 \mathrm{~Hz}$. Next, the frequency spectra were averaged within each condition. For each electrode, this resulted in one frequency spectrum per experimental condition and one additional spectrum for the baseline condition. Subsequently, the frequency spectra were combined spatially by averaging over electrodes to form the following electrode clusters (labels of the electrodes given in HydroCel GSN 130 terminology): frontal = 4, 5, 11, 12, 19, 20, 24, 28, 117, 118, 124; central $=\mathrm{Cz}, 7,30$, $31,36,37,55,80,87,104,105,106$; parietal $=52,53,60$, $61,62,67,72,77,78,85,86,92$; and occipital $=69,70,74$, $75,82,83,89$. From the spectra of these electrode clusters, the mean amplitude of the following frequency bands was extracted: theta $=4-7 \mathrm{~Hz}$, lower alpha $=$ $7.5-10 \mathrm{~Hz}$, upper alpha $=10.5-12.5 \mathrm{~Hz}$, lower beta $=13$ $-16 \mathrm{~Hz}$, and upper beta $=16.5-20 \mathrm{~Hz}$.

Following the spectral analysis, event-related synchronization (ERS) and event-related desynchronization (ERD) values were computed for the amplitudes in each frequency band and for each experimental condition [28,31]. The following formula was used for calculation:

$$
\mathrm{ERS} / \mathrm{ERD}=\frac{(\text { Amplitude in experimental condition }- \text { Amplitude in baseline condition }) \times 100}{\text { Amplitude in baseline condition }}
$$

$0.1-100 \mathrm{~Hz}$ was applied. Electrode impedance was maintained below $50 \mathrm{k} \Omega$.

The EEG data were preprocessed using Brain Vision Analyzer 2 software (version 2.1.1, http://www.brainpro ducts.com/downloads, Brain Products GmbH, Gilching, Germany). First, 20 electrodes from the outermost ring were excluded from further analysis because of their tendency to be affected by artifacts. Subsequently, EEG data were bandpass-filtered from 0.5 to $30 \mathrm{~Hz}$. Artifacts caused by eye blinks and horizontal eye movements were corrected using independent component analysis [30]. Artifact-contaminated electrodes were interpolated with the Hjorth nearest neighbor's method. The remaining artifacts were removed using an automatic raw data inspection. After preprocessing, an average reference was computed, and the data were segmented into segments of $4 \mathrm{~min}$ length corresponding to the experimental conditions and the
The ERS/ERD values characterize changes (\%) in the mean amplitude in the experimental conditions compared with the baseline condition. ERS/ERD values are relative values that have the advantage that unspecific inter-individual differences in amplitude are attenuated. The ERS/ERD values were subjected to subsequent statistical analysis.

\section{ECG data acquisition, preprocessing, and data analysis}

ECG data were collected using three electrodes (EL504; Biopac Systems, Goleta, California, USA), two of which were placed on the left and right forearm. A third electrode served as a ground electrode and was placed on the right elbow. The ECG data were sampled at $200 \mathrm{~Hz}$ (ECG100C; Biopac Systems) and recorded using AcqKnowledge software (version 4.4.2, http://www.biopac. com/product-category/research/software)). Brain Vision Analyzer 2 was used to segment the data into the experimental 
conditions and the baseline condition. Subsequently, the QRS complexes, more specifically the $\mathrm{R}$ peaks, were detected automatically in the raw ECG data. In addition, the data were visually inspected to ensure that no R peaks were missed by the automatic detection algorithm. The timing of the $\mathrm{R}$ peaks was extracted for further analysis.

Construction of the time series of intervals between successive R peaks (RR time series) and the calculation of HR and the heart rate variability (HRV) measure was performed using the R package RHRV (version 4.2.3, http://rhrv.r-forge. r-project.org/). The RR intervals were used to construct an RR time series. Next, outliers were removed automatically using default RHRV settings. Finally, the RR time series was interpolated to construct an equidistantly sampled time series. From the RR time series, HR (i.e. mean beats/min) and the power in the high-frequency band (HF-HRV: $0.15-0.4 \mathrm{~Hz}$ ) were computed. Both HR measures were then transformed using the natural logarithm. We did not include the low-frequency (LF-HRV: $0.04-0.15 \mathrm{~Hz}$ ) or very lowfrequency components of HRV (VLF-HRV: $\leq 0.04 \mathrm{~Hz}$ ) because their interpretation as indicators of autonomic activity still remains unclear [32]. Analogous to the EEG data analysis, we calculated the relative changes in the experimental conditions compared with the baseline condition. The following formula was used for HR and the HRV measure:

$$
\text { Change in HR/HRV measure }=\frac{(\mathrm{HR} / \mathrm{HRV} \text { in experimental condition }-\mathrm{HR} / \mathrm{HRV} \text { in baseline condition }) \times 100}{\mathrm{HR} / \mathrm{HRV} \text { in baseline condition }}
$$

\section{Statistical analysis}

All statistical analyses were carried out in $\mathrm{R}$ (version 3.3.2, http://wrww.R-project.org). For the analysis of the EEG data, we performed, separately for each frequency band, repeated-measures analysis of variance (rANOVA) with the ERS/ERD values as the dependent variable. In these analyses, the following factors were used: (i) Music $=$ Mozart versus Klangkarussell, (ii) Condition $=$ attentive versus passive, and (iii) Electrodes = frontal, central, parietal, and occipital. The rANOVAs were calculated using the $\mathrm{R}$ package ez (version 4.4-0, https://cran.r-project.org/web/packa ges/ez/index.html). For the analysis of HR and HF-HRV data, we applied the same analysis logic as for the above-mentioned EEG measures. Here, we calculated the rANOVAs with the following factors: (i) Music $=$ Mozart, Klangkarussell and (ii) Condition $=$ attentive, passive .

We will only discuss rANOVA results associated with a $P$ value less than or equal to 0.05 . These results are termed in our paper as significant, although we are fully aware of the fact that this term is critical in the context of experiments such as ours [33]. As we must take into consideration that $P$ values depend on sample size, we also calculated effect sizes. Effect sizes in the context of the rANOVAs are given using the generalized $\eta^{2}$ as recommended for a repeated-measures design [34]. A generalized $\eta^{2}$ more than 0.02 is considered a small effect, a generalized $\eta^{2}$ more than 0.13 is considered a moderate effect, and a generalized $\eta^{2}$ more than 0.26 is considered a large effect [34].

In addition to the rANOVAs, effect sizes as measured by Cohen's $d$ [35] were calculated for each EEG frequency band to investigate to what extent the ERS/ ERD values in the experimental conditions differed from 0 (using the $\mathrm{R}$ package 1sr, version 0.5, https:// cran.r-project.org/web/packages/lsr/index.html). Cohen's d measures were also calculated for the changes in the HR/HRV measure from baseline. In this context, a $d$ more than 0.2 is considered as small, a $d$ more than 0.5 is considered moderate, whereas a $d$ more than 0.8 is considered large.

\section{Results \\ Behavior}

The participants rated the musical pieces positive in all experimental conditions (valence ratings; KlangkarussellAttentive: mean $=4.9, \mathrm{SD}=0.92$, Klangkarussell-Passive: mean $=4.77, \mathrm{SD}=0.82$, Mozart-Attentive: mean $=4.97$, $\mathrm{SD}=0.85, \quad$ Mozart-Passive: mean $=4.8, \quad \mathrm{SD}=0.89$ ).
The participants also indicated moderately increased arousal in all experimental conditions (arousal ratings; KlangkarussellAttentive: mean $=3.93, \mathrm{SD}=1.34$, Klangkarussell-Passive: mean $=3.37, \quad \mathrm{SD}=1.35$, Mozart-Attentive: mean $=4.07$, $\mathrm{SD}=1.20$, Mozart-Passive: mean $=3.50, \mathrm{SD}=1.33$ ). There was no substantial difference between the valence and arousal ratings of the two musical pieces. The participants rated the subjectively experienced difficulty of the experimental conditions as follows: Klangkarussell-Attentive: mean $=3.53, \mathrm{SD}=1.5$, Klangkarussell-Passive: mean $=2.63$, $\mathrm{SD}=1.27, \quad$ Mozart-Attentive: mean $=4.23, \quad \mathrm{SD}=1.83$, Mozart-Passive: mean $=3.07, \mathrm{SD}=1.51$. The comparison of these subjective difficulty evaluations showed that the Mozart-Passive condition was evaluated as more difficult than the Klangkarussell-Passive condition (Wilcoxon signed-rank test, $P=0.048$ ). There was no difference between the Mozart-Attentive and the Klangkarussell-Attentive conditions in terms of subjective difficulty. Combining the difficulty evaluations for the two experimental conditions showed that listening to the Mozart musical piece was rated as more difficult and demanding than listening to the Klangkarussell piece (Wilcoxon signed-rank test, $P=0.01$ ). 
Fig. 1

\section{(a) ERS/ERD Klangkarussell-Attentive}
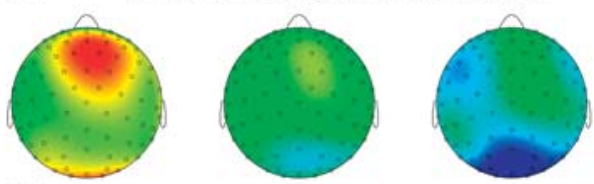

(b)

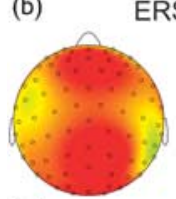

ERS/ERD Klangkarussell-Passive
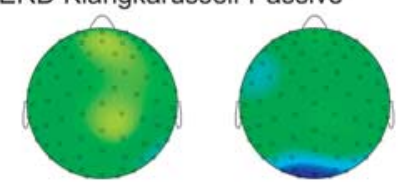

(c)

ERS/ERD Mozart-Attentive
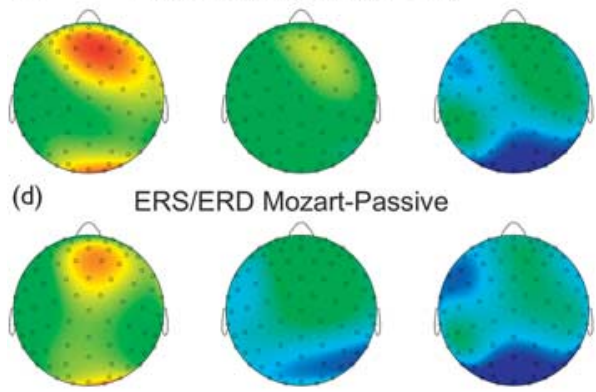

Theta

ERS/ERD Mozart-Passive

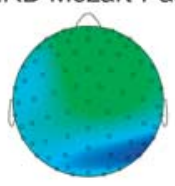

Lower Alpha

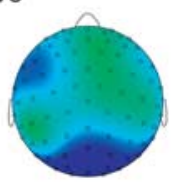

Upper Alpha
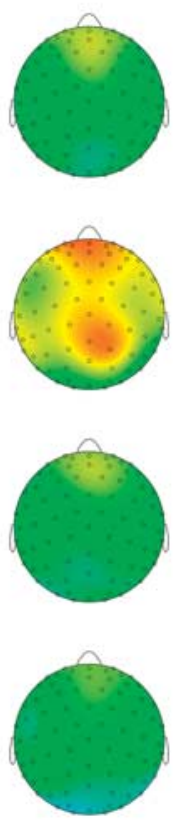

Lower Beta
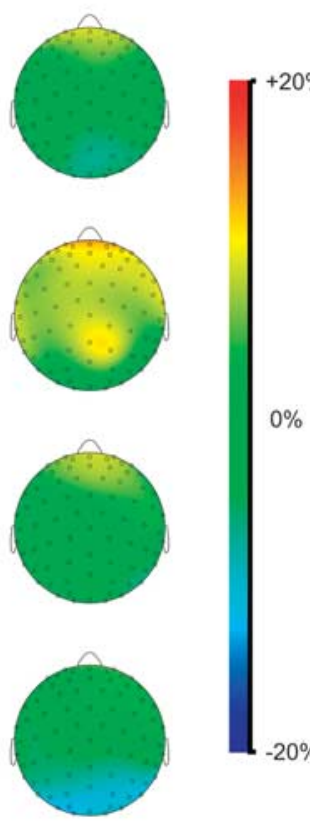

Upper Beta

Event-related synchronization (ERS) and event-related desynchronization (ERD) in the different experimental conditions and frequency bands. The hot colors indicate ERS and the cold colors indicate ERD. (a) ERS/ERD Klangkarussell-Attentive, (b) ERS/ERD Klangkarussell-Passive, (c) ERS/ERD Mozart-Attentive and (d) ERS/ERD Mozart-Passive.

\section{Event-related synchronization and event-related desynchronization}

Figure 1 shows the ERS/ERD results separately for each musical piece and experimental condition. As one can see from Fig. 1, oscillations in the theta frequency band strongly synchronize (ERS) in all experimental conditions, especially at frontal electrodes. For the KlangkarussellPassive condition, theta band ERS was observed at nearly all electrodes. For the lower alpha band, we found no or only very small ERS in all experimental conditions. In the Mozart-Passive condition, there were small lower alpha band ERDs at occipital electrodes. For the upper alpha band, several electrodes (especially at occipital positions) showed ERDs. For the lower and upper beta bands, we found ERS during the Klangkarussell-Passive condition at frontal electrode sites (lower and upper beta band).

Table 1 shows the effect sizes (Cohen's $d$ ) characterizing the changes in the EEG measures in the experimental conditions compared with baseline separately for each frequency band and electrode cluster.

In the following, the results of the rANOVAs of the ERS/ ERD values are described separately for each frequency band. A summary of the rANOVA results is shown in Table 2, which lists all $P$ values separately for each effect and frequency band. As recommended by Krauth [36] and consistent with the exploratory nature of our study,
Table 1 Summary of the effect sizes (Cohen's $d$ ) of the eventrelated synchronization and event-related desynchronization values for the different experimental conditions, frequency bands, and electrode clusters

\begin{tabular}{cccccc}
\hline & \multicolumn{5}{c}{ Frequency bands } \\
\cline { 2 - 6 } Condition & Theta & Lower alpha & Upper alpha & Lower beta & Upper beta \\
\hline \multirow{2}{*}{ Klangkarussell-Attentive } & & & & \\
F & +0.84 & +0.40 & -0.09 & +0.45 & +0.38 \\
$\mathrm{C}$ & +0.47 & +0.17 & -0.45 & +0.06 & -0.02 \\
$\mathrm{P}$ & +0.38 & -0.02 & -0.51 & -0.07 & -0.07 \\
$\mathrm{O}$ & $+\mathbf{0 . 6 1}$ & +0.15 & -0.28 & +0.06 & +0.09 \\
All & +0.56 & +0.16 & -0.31 & +0.10 & +0.08 \\
Klangkarussell-Passive & & & & \\
$\mathrm{F}$ & +0.86 & +0.51 & +0.22 & +0.61 & +0.52 \\
$\mathrm{C}$ & +0.84 & +0.35 & +0.12 & +0.49 & +0.35 \\
$\mathrm{P}$ & $+\mathbf{0 . 6 6}$ & +0.22 & -0.03 & +0.32 & +0.24 \\
$\mathrm{O}$ & +0.72 & +0.42 & 0.00 & +0.44 & +0.40 \\
All & +0.73 & +0.37 & +0.07 & +0.46 & +0.37 \\
Mozart-Attentive & & & & \\
$\mathrm{F}$ & +0.71 & +0.50 & -0.01 & +0.36 & +0.35 \\
$\mathrm{C}$ & +0.33 & +0.12 & -0.41 & -0.03 & -0.04 \\
$\mathrm{P}$ & +0.35 & 0.00 & -0.44 & -0.14 & -0.10 \\
$\mathrm{O}$ & +0.56 & +0.26 & -0.24 & +0.12 & +0.18 \\
All & +0.49 & +0.22 & -0.25 & +0.08 & +0.12 \\
Mozart-Passive & & & & \\
$\mathrm{F}$ & +0.51 & +0.19 & -0.05 & +0.30 & +0.22 \\
$\mathrm{C}$ & +0.33 & -0.10 & -0.32 & +0.04 & -0.08 \\
$\mathrm{P}$ & +0.37 & -0.20 & -0.45 & -0.09 & -0.26 \\
$\mathrm{O}$ & +0.64 & +0.07 & -0.38 & +0.06 & -0.02 \\
$\mathrm{All}$ & +0.47 & 0.00 & -0.29 & +0.08 & -0.02 \\
\hline
\end{tabular}

The direction of the effect is shown by the sign [plus $(+)=E R S$, minus $(-)=$ ERD]. Moderate and large effects are indicated in bold.

all, across all electrode clusters; C, central; ERS, event-related synchronization; ERD, event-related desynchronization; $F$, frontal; $\mathrm{P}$, parietal; $\mathrm{O}$, occipital. 
Table 2 Summary of the repeated-measures analysis of variance results broken down for each main effect and interaction, separately for each frequency band

\begin{tabular}{lccccc}
\hline & \multicolumn{5}{c}{ Frequency bands } \\
\cline { 2 - 6 } & Theta & $\begin{array}{c}\text { Lower } \\
\text { alpha }\end{array}$ & $\begin{array}{c}\text { Upper } \\
\text { alpha }\end{array}$ & $\begin{array}{c}\text { Lower } \\
\text { beta }\end{array}$ & $\begin{array}{c}\text { Upper } \\
\text { beta }\end{array}$ \\
\hline Condition & 0.16 & 0.69 & 0.26 & 0.16 & 0.42 \\
Music & $\mathbf{0 . 0 2}$ & 0.06 & 0.10 & $\mathbf{0 . 0 4}$ & 0.09 \\
Electrodes & $\mathbf{0 . 0 0 1}$ & $\mathbf{0 . 0 1}$ & $\mathbf{0 . 0 2}$ & $\mathbf{0 . 0 0 5}$ & $\mathbf{0 . 0 0 7}$ \\
Condition : Music & 0.07 & $\mathbf{0 . 0 2}$ & $\mathbf{0 . 0 4}$ & $\mathbf{0 . 0 3}$ & $\mathbf{0 . 0 2}$ \\
Condition : Electrodes & 0.43 & 0.94 & 0.83 & 0.87 & 0.76 \\
Music : Electrodes & 0.69 & 0.90 & 0.85 & 0.70 & 0.68 \\
Condition : Music : Electrodes & 0.31 & 0.41 & 0.90 & 0.84 & 0.48 \\
\hline
\end{tabular}

The $P$ values for each main effect and interaction are shown. Effects associated with $P \leq 0.05$ are indicated in bold letters.

we report all $P$ values to provide the reader with complete information on our analyses.

The rANOVA for the theta frequency band showed significant main effects for the factors Music $[F(1,29)=6.24$, $P=0.02$, generalized $\left.\eta^{2}=0.02\right]$ and Electrodes $[F(3,87)=$ 6.44, $P<0.001$, generalized $\left.\eta^{2}=0.03\right]$. The main effect for Music is driven by larger ERS during the KlangkarussellPassive condition (see Fig. 2a). The main effect for Electrodes is driven by larger ERS values at posterior electrodes (see Fig. 3a). No further main effect or interactions were significant. In Fig. 2a, the ERS values for the theta band are shown broken down for the four conditions. As shown in Fig. 2a, the theta band synchronizes much stronger during the Klangkarussell-Passive condition than during the other three conditions. During the Klangkarussell-Passive condition, there was strong ERS as shown by a moderate effect size $(d=0.73)$ compared with the ERS values during the other conditions, which showed small or moderate effect sizes $(d=0.47-0.56)$.

For the lower alpha frequency band, we found a significant main effect for the factor Electrodes $[F(3,87)=3.82$, $P=0.01$, generalized $\left.\eta^{2}=0.02\right]$ and a significant interaction between the factors Condition and Music $[F(1,29)=5.91$, $P=0.02$, generalized $\left.\eta^{2}=0.01\right]$. We found no further main effects or interactions. As shown in Fig. 2b, the Condition $\times$ Music interaction is driven by a large ERS during the Klangkarussell-Passive condition in addition to a lack of change from baseline in the Mozart-Passive condition. The significant effect for the factor Electrodes is driven by ERDs at parietal electrodes (see Fig. 3b).

In the upper alpha frequency band, the rANOVA showed a significant main effect for the factor Electrodes $\left[F(3,87)=3.55, P=0.02\right.$, generalized $\left.\eta^{2}=0.02\right]$ and a significant Condition $\times$ Music interaction $[F(1,29)=4.72$, $P=0.04$, generalized $\left.\eta^{2}=0.01\right]$. As shown in Fig. 2c, the Condition $\times$ Music interaction is characterized by ERDs in three experimental conditions (Klangkarussell-Attentive, Mozart-Attentive, and Mozart-Passive). In the KlangkarussellPassive condition, there was no change from baseline in either direction (i.e. no ERS or ERD). The significant effect for the factor Electrodes is mainly driven by ERDs at posterior electrodes (see Fig. 3c). No further main effects or interactions were found.

In the lower beta frequency band, we found significant main effects for the factor Music $[F(1,29)=4.62, P=0.04$, generalized $\left.\eta^{2}=0.02\right]$, for the factor Electrodes $[F(3,87)=$ $4.54, P=0.005$, generalized $\left.\eta^{2}=0.02\right]$, and a significant Condition $\times$ Music interaction $[F(1,29)=5.01, P=0.03$, generalized $\left.\eta^{2}=0.02\right]$. The Condition $\times$ Music interaction is driven by a substantial ERS in the KlangkarussellPassive condition (see Fig. 2d). The significant effect for the factor Electrodes is mainly driven by ERSs at frontal and central electrodes (see Fig. 3d). There were no further significant main effects or interactions.

Finally, in the upper beta frequency band, we found a main effect for the factor Electrodes $[F(3,87)=4.34, P=0.01$, generalized $\left.\eta^{2}=0.02\right]$ and an interaction between the factors Condition and Music $[F(1,29)=6.58, P=0.02$, generalized $\left.\eta^{2}=0.02\right]$. No further main effect or interactions were significant. The Condition $\times$ Music interaction is driven by a large ERS during the Klangkarussell-Passive condition (see Fig. 2e). The significant effect for the factor Electrodes is mainly driven by ERSs at frontal electrodes and ERDs at parietal electrodes (see Fig. 3e). Overall, the pattern was similar compared with the lower beta band.

\section{Heart rate and heart rate variability}

Table 3 lists the effect sizes (Cohen's $d$ ) describing the HR/HRV measure changes in each experimental condition in comparison with the baseline condition. There were small HR increases (as indicated by Cohen's $d$ ) relative to baseline in the Klangkarussell conditions and a moderate change in the Mozart-Attentive condition. In the Mozart-Passive condition, there was no HR change. The HF-HRV measure showed strong decreases from baseline in all experimental conditions, with the strongest changes during the Klangkarussell-Passive and the Mozart-Attentive conditions.

The rANOVA for the HR showed no significant main effects for the factors Condition and Music, and no significant interaction. The rANOVA for the HF-HRV showed a significant main effect for Condition $[F(1,29)=4.28, P=0.047$, generalized $\eta^{2}=0.1$, which is driven by slightly larger power decreases during attentive music listening (see Fig. 4).

\section{Discussion}

The most important finding of this exploratory study is that the same musical piece can evoke entirely different neural activations depending on the direction of attention. This attention-effect was mainly present for the electronic musical piece by Klangkarussell, but not for the classical musical piece by Mozart. For the Klangkarussell musical piece, we consistently found stronger ERS during the passive listening condition than during the attentive listening condition. This effect was 

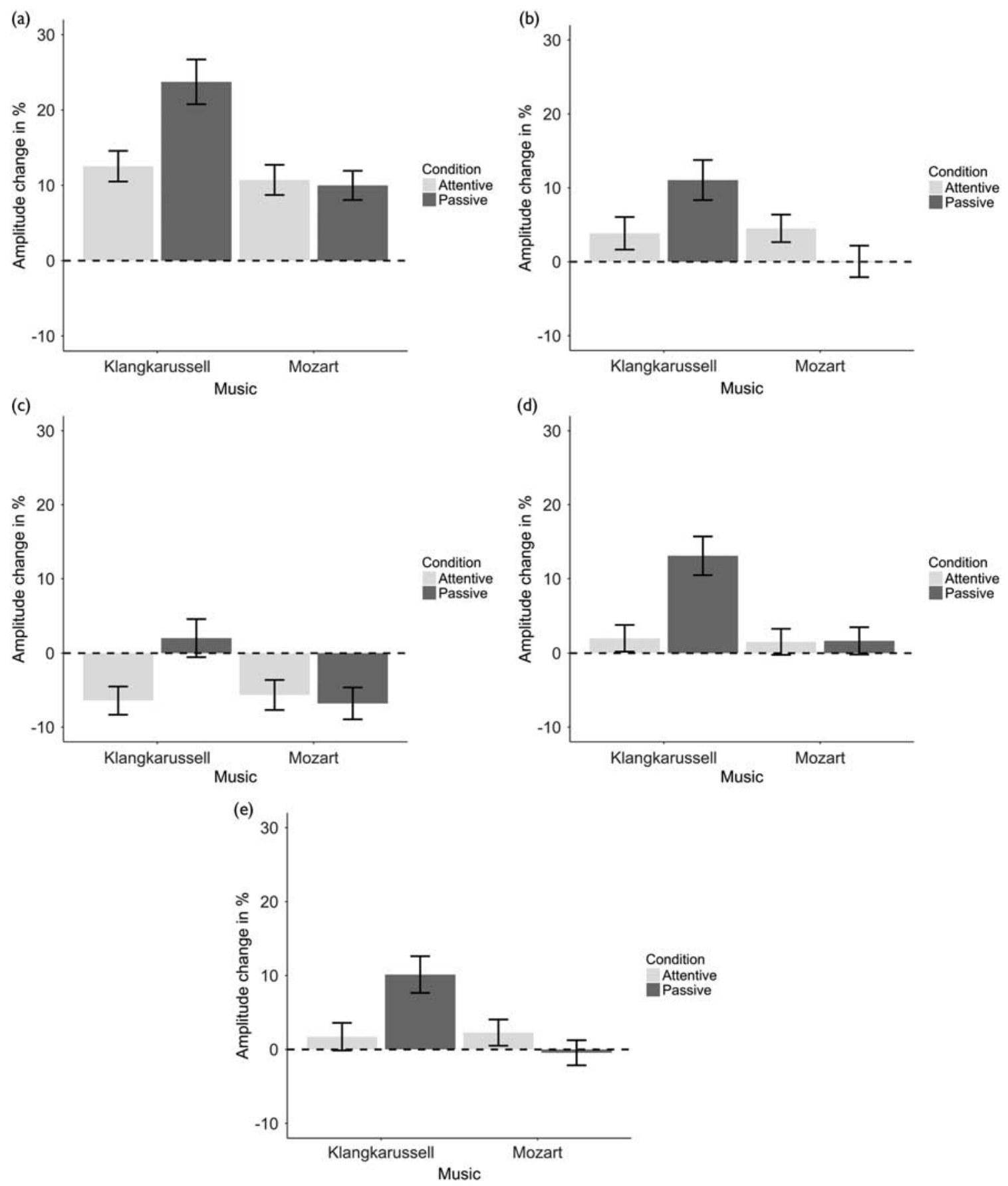

Event-related synchronization/event-related desynchronization separately for each frequency band. (a) Theta, (b) lower alpha, (c) upper alpha, (d) lower beta, (e) upper beta. Error bars visualize the between-subject SEM.

present in all investigated frequency bands. For the Mozart piece, we only identified small or absent ERS values in nearly all frequency bands. In the upper alpha band, we identified small to moderately strong ERD values for the Mozart piece during both attentive conditions. The HF-HRV measure showed strong decreases compared with baseline during all conditions, indicating a strong inhibition of the parasympathetic and an activation of the sympathetic nervous system. HF-HRV changes were stronger for the Mozart-Attentive compared with the Mozart-Passive condition.

The uncovered attention-dependent EEG response for the Klangkarussell-Passive listening condition resembles 

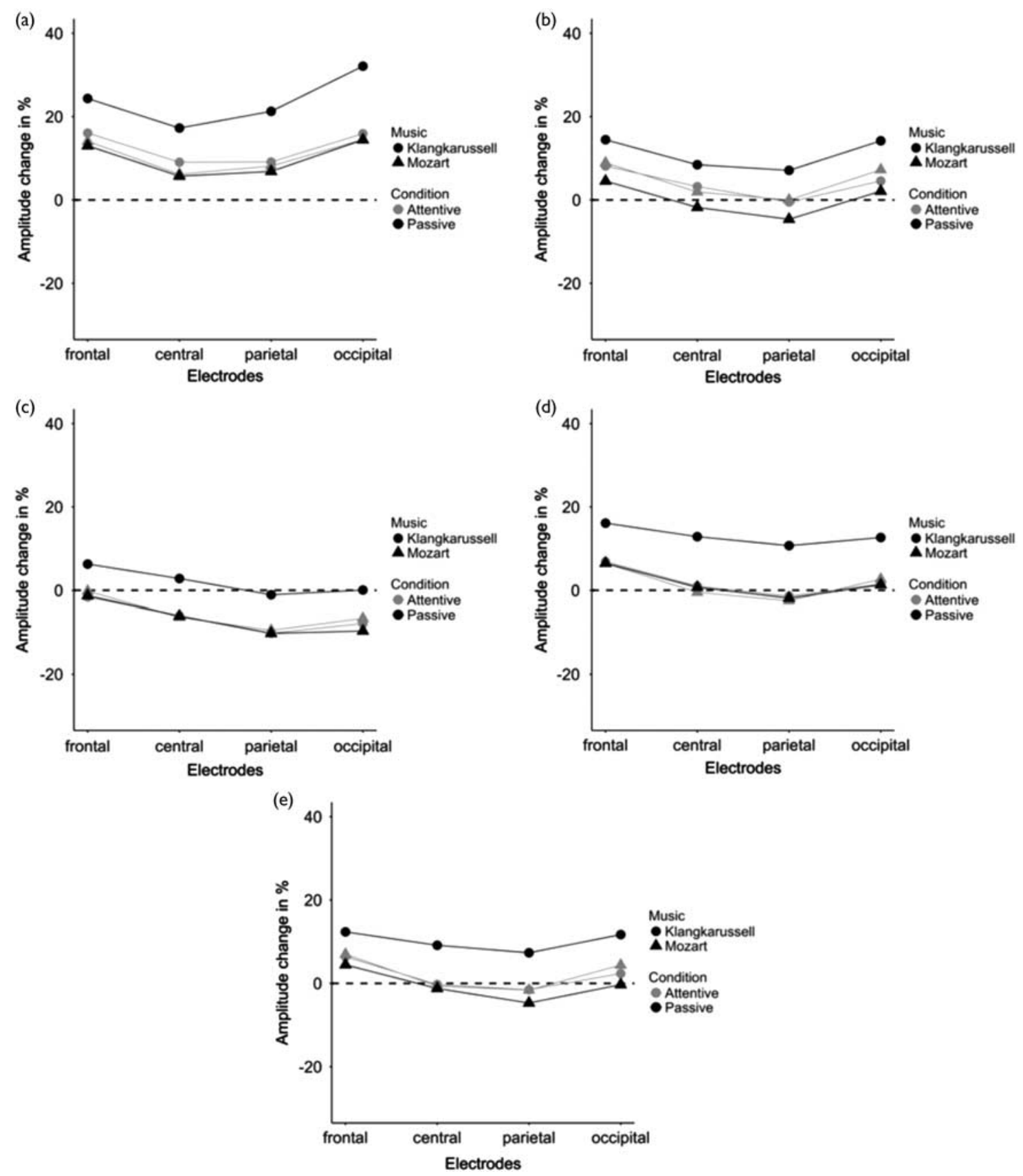

Event-related synchronization/event-related desynchronization separately for each frequency band and electrode cluster. (a) Theta, (b) lower alpha, (c) upper alpha, (d) lower beta, (e) upper beta.

the synchronizations across all frequency bands found in a previous study of our group [37]. In this earlier study, we found a general EEG synchronization while the participants listened to a famous and well-appreciated aria. We have interpreted this synchronization pattern as a neurophysiological state associated with increased internal but reduced external attention. This state is accompanied by increased inhibition of brain networks not involved in generating this internal state. We have speculated that this state would fit with a psychological state in which the listeners are 'drawn into' the musical piece. A similar, or even the same, state could have been induced during the Klangkarussell-Passive condition. This particular musical piece was well appreciated by the participants (most of them listen more to pop music than to classical music) and was a major hit during the time of 
testing. Thus, this musical piece might have required fewer attentional resources, and therefore, the participants could have listened in a 'by-the-way style' that did not affect the performance on the primary task (here: counting the occurrence of certain aspects of the film sequences). This interpretation also fits with the subjective assessment of the experienced difficulty by the participants during the two conditions. The participants rated the Mozart-Passive condition as more demanding and difficult than the Klangkarussell-Passive condition. Therefore, it is quite plausible that the KlangkarussellPassive condition provides more of an opportunity to get into this psychological state.

The Mozart musical piece, in contrast, was associated with small to moderate ERDs in the upper alpha band during both listening conditions, particularly at posterior electrodes. This ERD of the upper alpha band indicates an activation of the dorsal attention network (comprising the superior parietal lobule, the intraparietal sulcus area, and parts of the dorsal visual cortex), which is most prominently involved in selective spatial attention [38]. Perhaps the participants directed their attention more strongly to the film sequence and were not drawn into the Mozart musical piece. In other words, the classical

Table 3 Effect sizes as measured by Cohen's $d$ describing changes in the heart rate/heart rate variability measure in the experimental conditions in comparison with the baseline condition

\begin{tabular}{lcc}
\hline Condition & HR & HF-HRV \\
\hline Klangkarussell-Attentive & +0.29 & -0.94 \\
Klangkarussell-Passive & +0.29 & -1.31 \\
Mozart-Attentive & +0.37 & -1.32 \\
Mozart-Passive & -0.03 & -0.92 \\
\hline
\end{tabular}

The direction of the effect is indicated by the sign [plus $(+)=$ increase, minus $(-)=$ decrease .

$\mathrm{HR}$, heart rate; HF-HRV, high-frequency heart rate variability. musical piece did not induce the particular neurophysiological and psychological state induced by the Klangkarussell musical piece.

However, it should be kept in mind that the listening conditions used in this experiment are different compared with the listening condition used in the aforementioned study. In the earlier study, the participants' listening to the musical piece was unrestricted, while the listening conditions in this study are more of a dual-task situation. Therefore, one might also interpret the present findings in the context of the dual-task literature. Power increases in the theta and beta bands (partly with decreases in the upper alpha band) have frequently been reported in the dual-task literature [39-41] and with increased mental workload $[42,43]$. Increased activity in the alpha band (especially over parietal regions) is often associated with increased activity of the tonic alertness network (comprising the dorsal anterior cingulum, the anterior insula, the thalamus, and the anterior prefrontal cortex) [38]. Thus, in the context of these findings, passive listening to the Klangkarussell musical piece would have induced an increased workload and more tonic alertness, requiring increased activation and involvement of the responsible neural networks. However, this interpretation seems to be less plausible as the ANS responses indicate ANS arousal for all conditions. If mental workload was increased during one of these listening conditions, we would have anticipated stronger HF-HRV responses as well as stronger HR increases $[44,45]$. In summary, mental workload is a less plausible explanation for this pattern of EEG and ANS responses. We favor the hypothesis that the music by Klangkarussell had the power to induce a specific psychological and neurophysiological state that comes close to what we have described as being 'drawn into the music'.
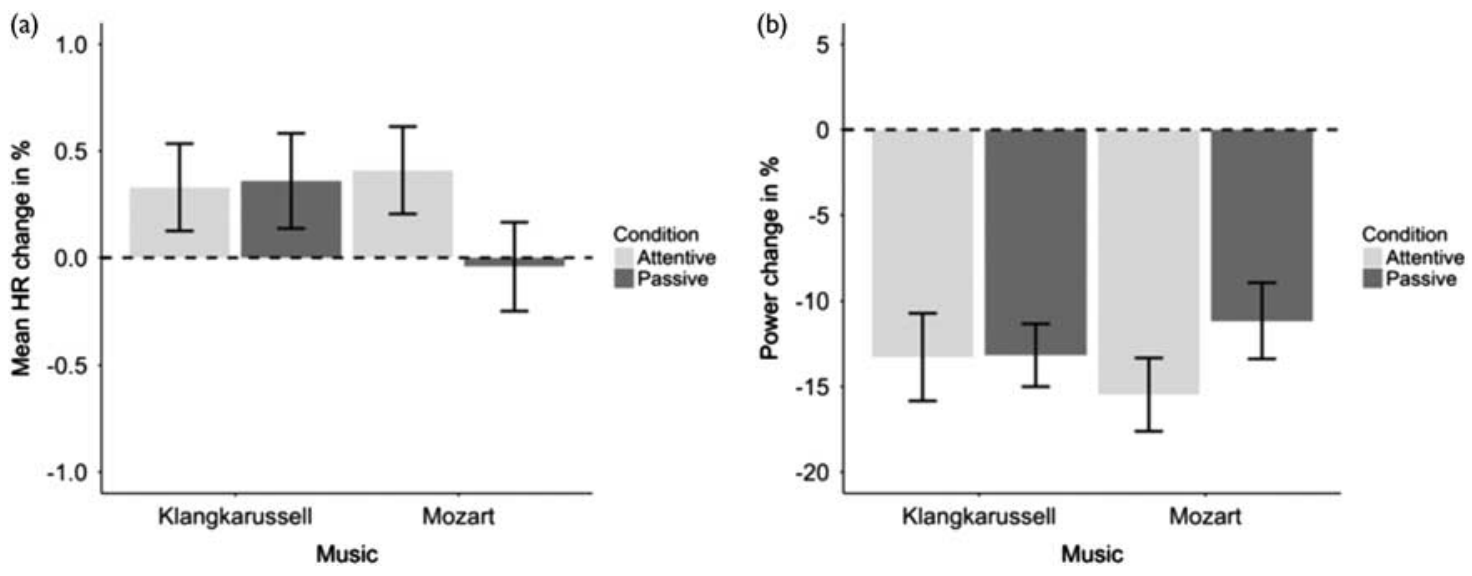

Heart rate (HR) and high-frequency heart rate variability (HF-HRV). (a) HR changes relative to baseline in the experimental conditions. (b) High-frequency HF-HRV changes relative to baseline in the experimental conditions. Error bars represent the between-subject SEM. 
In this study, we refrained from estimating intracortical sources on the basis of surface EEG signals to base our interpretation on more robust EEG data. However, from functional MRI studies it can be seen that directing attention away from the auditory stimuli results in less and more variable auditory cortex activation. Therefore, we hypothesize that in the passive music listening conditions, auditory cortex activation may have decreased, whereas activation in the visual areas may have increased. However, this auditory attention-effect has to be shown in future experiments using musical stimuli $[23,24]$.

Although the results of our study must be substantiated by follow-up experiments using more participants, different musical pieces, and further experimental manipulations, we would like to emphasize that more experiments should be conducted using similar paradigms to the one used in this study. Future experiments should study neurophysiological and autonomic responses to music in situations resembling everyday situations. When we listen to music in everyday situations, we often simultaneously perform other tasks. In the present experiment, we have attempted to model such an everyday situation to study the EEG and ANS responses to music in a more ecologically valid setting. By doing this, we have shown that the neurophysiological responses to a particular musical piece strongly depend on the attention focus. Thus, we have provided scientific arguments for studying the neural underpinnings of music perception in more ecologically valid experiments.

\section{Acknowledgements}

This research was supported by the Swiss National Foundation (grant no. 320030_163149 granted to L.J.).

\section{Conflicts of interest}

There are no conflicts of interest.

\section{References}

1 Baumgartner T, Lutz K, Schmidt CF, Jäncke L. The emotional power of music: how music enhances the feeling of affective pictures. Brain Res 2006; 1075:151-164.

2 Koelsch S, Fritz T, V Cramon DY, Müller K, Friederici AD. Investigating emotion with music: an fMRI study. Hum Brain Mapp 2006; 27:239-250.

3 Koelsch S, Skouras S, Fritz T, Herrera P, Bonhage C, Küssner MB, et al. The roles of superficial amygdala and auditory cortex in music-evoked fear and joy. Neuroimage 2013; 81:49-60.

4 Salimpoor VN, van den Bosch I, Kovacevic N, Mclntosh AR, Dagher A, Zatorre RJ. Interactions between the nucleus accumbens and auditory cortices predict music reward value. Science 2013; 340:216-219.

5 Salimpoor VN, Zald DH, Zatorre RJ, Dagher A, Mclntosh AR. Predictions and the brain: how musical sounds become rewarding. Trends Cogn Sci 2015; 19:86-91.

6 Trost W, Ethofer T, Zentner M, Vuilleumier P. Mapping aesthetic musical emotions in the brain. Cereb Cortex 2012; 22:2769-2783.

7 Trost W, Frühholz S, Cochrane T, Cojan Y, Vuilleumier P. Temporal dynamics of musical emotions examined through intersubject synchrony of brain activity. Soc Cogn Affect Neurosci 2015; 10:1705-1721.

8 Jäncke L. The dynamic audio-motor system in pianists. Ann NY Acad Sci 2012; 1252:246-252.

9 Jäncke L, Alahmadi N. Detection of independent functional networks during music listening using electroencephalogram and sLORETA-ICA. Neuroreport 2016; 27:455-461.
10 Rogenmoser L, Zollinger N, Elmer S, Jäncke L. Independent component processes underlying emotions during natural music listening. Soc Cogn Affect Neurosci 2016; 11:1428-1439.

11 Altenmüller E, Schürmann K, Lim VK, Parlitz D. Hits to the left, flops to the right: different emotions during listening to music are reflected in cortical lateralisation patterns. Neuropsychologia 2002; 40:2242-2256.

12 Bhattacharya J, Petsche H. Universality in the brain while listening to music. Proc R Soc Lond B Biol Sci 2001; 268:2423-2433.

13 Bhattacharya J, Petsche $\mathrm{H}$. Enhanced phase synchrony in the electroencephalograph gamma band for musicians while listening to music. Phys Rev E Stat Nonlin Soft Matter Phys 2001; 64:012902.

14 Bhattacharya J, Petsche $\mathrm{H}$. Musicians and the gamma band: a secret affair? Neuroreport 2001; 12:371-374.

15 Bhattacharya J, Petsche H, Pereda E. Long-range synchrony in the gamma band: role in music perception. J Neurosci 2001; 21:6329-6337.

16 Carrus E, Koelsch S, Bhattacharya J. Shadows of music-language interaction on low frequency brain oscillatory patterns. Brain Lang 2011; 119:50-57.

17 Mikutta C, Altorfer A, Strik W, Koenig T. Emotions, arousal, and frontal alpha rhythm asymmetry during Beethoven's 5th symphony. Brain Topogr 2012; 25:423-430.

18 Mikutta CA, Maissen G, Altorfer A, Strik W, Koenig T. Professional musicians listen differently to music. Neuroscience 2014; 268:102-111.

19 Sarnthein J, vonStein A, Rappelsberger P, Petsche H, Rauscher FH, Shaw GL. Persistent patterns of brain activity: an EEG coherence study of the positive effect of music on spatial-temporal reasoning. Neurol Res 1997; 19:107-116.

20 Tsang CD, Trainor LJ, Santesso DL, Tasker SL, Schmidt LA. Frontal EEG responses as a function of affective musical features. Ann NYAcad Sci 2001; 930:439-442.

21 Schaefer RS, Vlek RJ, Desain P. Music perception and imagery in EEG: alpha band effects of task and stimulus. Int J Psychophysiol 2011; 82:254-259.

22 Schaefer RS, Desain P, Farquhar J. Shared processing of perception and imagery of music in decomposed EEG. Neuroimage 2013; 70:317-326.

23 Jäncke L, Mirzazade S, Shah NJ. Attention modulates activity in the primary and the secondary auditory cortex: a functional magnetic resonance imaging study in human subjects. Neurosci Lett 1999; 266:125-128.

24 Jäncke L, Buchanan TW, Lutz K, Shah NJ. Focused and nonfocused attention in verbal and emotional dichotic listening: an FMRI study. Brain Lang 2001; 78:349-363.

25 Johnson JA, Zatorre RJ. Attention to simultaneous unrelated auditory and visual events: Behavioral and neural correlates. Cereb Cortex 2005; 15:1609-1620.

26 Petkov Cl, Kang X, Alho K, Bertrand O, Yund EW, Woods DL. Attentional modulation of human auditory cortex. Nat Neurosci 2004; 7:658-663.

27 Hutcherson CA, Goldin PR, Ochsner KN, Gabrieli JD, Barrett LF, Gross JJ. Attention and emotion: does rating emotion alter neural responses to amusing and sad films? Neuroimage 2005; 27:656-668.

28 Markovic A, Kühnis J, Jäncke L. Task context influences brain activation during music listening. Front Hum Neurosci 2017; 11:342.

29 Annett M. A classification of hand preference by association analysis. $\mathrm{Br} J$ Psychol 1970; 61:303-321.

30 Jung TP, Makeig S, Humphries C, Lee TW, McKeown MJ, Iragui V, et al. Removing electroencephalographic artifacts by blind source separation. Psychophysiology 2000; 37:163-178.

31 Pfurtscheller G, Lopes da Silva FH. Event-related EEG/MEG synchronization and desynchronization: basic principles. Clin Neurophysiol 1999; 110:1842-1857.

32 Billman GE. Heart rate variability - a historical perspective. Front Physiol $2011 ; 2: 86$

33 Benjamin DJ, Berger JO, Johannesson M, Nosek BA, Wagenmakers EJ, Berk R, et al. Redefine statistical significance. Nat Hum Behav 2017; 2:6-10.

34 Bakeman R. Recommended effect size statistics for repeated measures designs. Behav Res Methods 2005; 37:379-384.

35 Cohen J. Statistical power analysis for the behavioral sciences. New York, NY: Routledge; 1988.

36 Krauth J. Distribution-free statistics An application-oriented approach. Amsterdam; New York; Oxford: Elsevier; 1988.

37 Jäncke L, Kühnis J, Rogenmoser L, Elmer S. Time course of EEG oscillations during repeated listening of a well-known aria. Front Hum Neurosci 2015; 9:401.

38 Sadaghiani S, Scheeringa R, Lehongre K, Morillon B, Giraud AL, Kleinschmidt A. Intrinsic connectivity networks, alpha oscillations, and tonic alertness: a simultaneous electroencephalography/functional magnetic resonance imaging study. J Neurosci 2010; 30:10243-10250. 
39 Casutt G, Martin M, Jäncke L. Driving simulator training is associated with reduced inhibitory workload in older drivers. Geriatrics 2016; 1:16.

40 Lin CT, Chen SA, Chiu TT, Lin HZ, Ko LW. Spatial and temporal EEG dynamics of dual-task driving performance. J Neuroeng Rehabil 2011; 8:11.

41 Pizzamiglio S, Naeem U, Abdalla H, Turner DL. Neural correlates of singleand dual-task walking in the real world. Front Hum Neurosci 2017; 11:460.

42 Gevins A, Leong H, Du R, Smith ME, Le J, DuRousseau D, et al. Towards measurement of brain function in operational environments. Biol Psychol 1995; 40:169-186.
43 Gevins A, Smith ME, Leong H, McEvoy L, Whitfield S, Du R, et al. Monitoring working memory load during computer-based tasks with EEG pattern recognition methods. Hum Factors 1998; 40:79-91.

44 Koelsch S, Jäncke L. Music and the heart. Eur Heart J 2015; 36:3043-3049.

45 Thayer JF, Lane RD. Claude Bernard and the heart-brain connection: further elaboration of a model of neurovisceral integration. Neurosci Biobehav Rev 2009; 33:81-88. 